\title{
There and back again: Putting the vectorial movement planning hypothesis to a critical test
}

Based on psychophysical evidence about how learning of visuomotor transformation generalizes, it has been suggested that movements are planned on the basis of movement direction and magnitude, i.e. the vector connecting movement origin and targets. This notion is also known under the term "vectorial planning hypothesis". Previous psychophysical studies, however, have included separate areas of the workspace for training movements and testing the learning. This study eliminates this confounding factor by investigating the transfer of learning from forward to backward movements in a center-out-and-back task, in which the workspace for both movements is completely identical. Visual feedback allowed for learning only during movements towards the target (forward movements) and not while moving back to the origin (backward movements). When subjects learned the visuomotor rotation in forward movements, initial directional errors in backward movements also decreased to some degree. This learning effect in backward movements occurred predominantly when backward movements featured the same movement directions as the ones trained in forward movements (i.e., when opposite targets were presented). This suggests that learning was transferred in a direction specific way, supporting the notion that movement direction is the most prominent parameter used for motor planning. 


\section{Authors and Affiliations}

2 Eva-Maria Kobak ${ }^{1,3}$, Simone Cardoso de Oliveira ${ }^{1,2}$

31 Bernstein Center Freiburg, University of Freiburg, Germany

42 BrainLinks-BrainTools, Cluster of Excellence, University of Freiburg, Germany

53 Department of Bioengineering, Imperial College London, United Kingdom

6 Corresponding author: Eva-Maria Kobak, email: eva-maria.kobak10@alumni.imperial.ac.uk

\section{Introduction}

8 An approach frequently used to investigate the functional organization of the motor system is to

9 study learning of visuomotor transformations and the transfer of such learning to untrained

10 conditions. For example, visuomotor rotations have frequently been applied while subjects

11 perform simple reaching movements. Initially, the transformation leads to movement errors

12 reflecting the magnitude of the rotation, but gradually, most subjects are able to adapt to the

13 rotation. For this to happen, the internal representation of the movement has to be changed

14 (Imamizu, Uno \& Kawato, 1995). There are several ways how this may be achieved: Remapping

15 of the locations of origins and targets ("position remapping") is one possibility. Another is that

16 subjects remember the posture assumed when successfully reaching the target, based on the idea

17 that movements are planned by converging to a final end posture (Polit \& Bizzi, 1978,

18 Rosenbaum et al., 1995). A third possibility is that the alteration of movement direction to a given

19 target is remembered, based on the idea that movements are planned on the basis of the vector

20 connecting the starting location to the target ("vectorial planning" Gordon, Ghilardi \& Ghez,

21 1994).

22 While some early studies provided evidence supporting the idea of final end posture being

23 assumed by subjects (Polit \& Bizzi, 1978, Rosenbaum, Meulenbroek \& Vaughan, 1999), other

24 evidence points more towards the vectorial planning hypothesis (Gordon, Ghilardi \& Ghez, 1994;

25 Vindras et al., 1998; Messier \& Kalaska, 1999; Krakauer et al., 2000; Krakauer, 2009).

26 When visuomotor rotations were trained, learning was found to be transferred only to movements

27 in the same direction as the trained one, and not to previously trained targets that were

28 approached from other directions (Krakauer et al., 2000, Wang \& Sainburg, 2005). These studies, 
29 however, used different areas of the workspace for learning and test trials, and therefore

30 introduced space as a confounding variable to the experiments. In the Krakauer et al. (2000)

31 study, test trials started at the same origin, but were aimed at targets that had not been visited

32 during learning. In the Wang \& Sainburg (2005) study, the starting positions for test trials were in

33 completely different regions of the workspace, which were not visited during learning at all. The

34 transfer of learning that both studies found for test targets might as well be due to a position

35 remapping learning effect that remained restricted to the trained workspace as to a transfer of the

36 learned direction.

37 To test this possibility, we applied a revised version of the center-out-and-back task, making use

38 of the fact that backward movements occur in exactly the same location of the workspace as

39 forward movements. Our task provided visual feedback about hand position only during forward

40 movements. During backward movements, the cursor denoting hand position was not visible.

41 Thus, learning could occur only in forward movements, and backward movements could be used

42 for testing of learning transfer. This experiment constitutes an essential test of the vectorial

43 planning hypothesis, the important factor being that position in workspace is not added as a

44 confounding variable (see Figure 1).

45 Forward and backward movements in our experiment were separated by a short break at the

46 target, rendering forward and backward movements clearly distinguishable. Although Krakauer et

47 al. (2000) also used a center-out-and-back paradigm in their experiments, they did not include

48 any detailed analysis of backward movements. This may partly be due to the fact that their task

49 did not require subjects to stop at the target, making backward movements not very well

50 distinguishable from forward movements. Also, in their task, visual feedback was present

51 throughout the task.

52 To put the hypothesis of vectorial planning to a crucial test, we designed three different variants

53 of the center-out-and-back paradigm. In all variants, a 60 degrees visuomotor rotation was

54 applied in the learning trials. In the first variant (paradigm one $=\mathrm{P} 1$ ), subjects moved to and from

5512 targets distributed on a circle around the central starting position, such that learning during

56 forward movements included movement directions from 0 to 360 degrees in 30 degree

57 increments. In the second (paradigm two $=\mathrm{P} 2$ ) and third (paradigm three $=\mathrm{P} 3$ ) variant,

58 movements were directed only to two targets, either $180(\mathrm{P} 2)$ or 60 degrees (P3) apart from each

59 other. This means that in $\mathrm{P} 2$, the directions of forward movements to one target were equal to

60 backward movements from the other target, while in P3, backward and forward movement

61 directions did not match. 
62 If subjects would learn the shifted locations of the target and would make use of this knowledge

63 when planning backward movements, transfer of learning from forward to backward movements

64 would occur in all three variants of the paradigm, supporting the position remapping theory. If

65 learning would be based on learning the rotated directions, however, backward movements

66 should only be affected if they would occur in the directions that were trained during forward

67 movements, i.e., in experiments one and two. This would be in accordance with the vectorial

68 movement planning hypothesis (Figure 2).

69 We found that transfer of learning from forward to backward movements preferentially occurred

70 in paradigms one and two. Thus, our study further supports the vectorial planning hypothesis and

71 strengthens the idea that movement direction rather than the locations of origin and target is the

72 most prominent parameter used by the motor system for planning movements.

\section{Materials and Methods}

\section{Experimental Setup}

75 A phantom device 1.5 HF (SensAble technologies, Woburn MA, USA) was used to track 76 subjects' movements. The resulting trajectories are shown in Figure 3. The device was programmed to move frictionlessly in a horizontal plane directly under a horizontal board in front of which subjects were seated. During the experiment, the momentary position of the Phantom handle end point was recorded, digitized, visualized on a computer monitor and projected onto the board in such a way that projection and actual position of the phantom end-point were vertically aligned (in the non-rotated condition, see Figure 1A). The sampling rate for the position recording was $100 \mathrm{~Hz}$, and the gain with respect to the real movements was set to one, i.e., cursor movements had the same amplitudes as hand movements. The phantom device was

84 programmed to autonomously move back to the centre of the horizontal workspace after each 85 trial.

\section{Participants}

87 All subjects participating in the experiment were right-handed (verified by a modified Edinburgh Handedness Inventory, (Oldfield, 1971)) and naive as to the purpose of the study. Experimental procedures were approved by the local ethics committee (University of Freiburg), and all participants gave their informed written consent prior to starting the experiment. In total, 42 
91 subjects were tested in the experiment. Thirteen of these had to be excluded from analysis since

92 they failed to learn the visuomotor rotation. Successful learning was defined as a significant

93 difference between the first and last 50 movement errors under the rotation (irrespective of target

94 location, see below), using a standard t-test with $\mathrm{p}<0.05$. As we wanted to test the transfer of

95 learning from forward to backward movements, it was necessary to exclude subjects who did not

96 show any learning in the forward movements. Of the remaining subjects, 9 were tested in the

97 first, and 10 each in the second and third paradigm described in the following paragraph (see also

98 Figure 1B).

99 Paradigms and Trial-Sequence

100 All subjects participating in our experiment were assigned to one of three experimental groups,

101 each of which was tested in a different paradigm. Subjects in all three groups had to complete

102 three experimental blocks in which they had to perform out-and-back reaching movements.

103 Importantly, visual feedback for their movements was only given during forward movements and

104 switched off when subjects returned to the origin. In the first block (familiarization block,

105 consisting of 120 trials), subjects got veridical visual feedback about their movements. This block

106 was needed to verify that, given non-rotated visual feedback during forward movements, subjects

107 would move straight to the target and back to origin, even if during backward movements, visual

108 feedback was absent. In the second block (learning block, consisting of 360 trials), a visuomotor

109 rotation (60 degrees around the origin of movements) was introduced during forward movements.

110 In the third block (washout block, consisting of 120 trials), veridical, non-rotated cursor feedback

111 was given again during the forward movements.

112 The three paradigms were set up as follows: The first group of subjects was tested in a paradigm

113 in which one of 12 targets was presented in each trial (P1 - targets were chosen pseudo-randomly

114 over each block). The 12 targets were equidistantly distributed (i.e. 30 degrees apart from each

115 other) on a circle with radius $5 \mathrm{~cm}$ around the movement origin (Figure 1B P1). The second

116 group of subjects was tested in a paradigm in which one of only two targets was presented in

117 each trial (P2). The two targets were positioned at 60 and 240 degrees from the origin (0 degrees

118 meaning rightward, 90 degrees meaning forward direction, seen from the perspective of

119 participants, see Figure 1 B, P2), i.e. at 180 degrees from each other. In the figures depicting the

120 targets separately, these targets will be referred to as P2a (at 60 degrees) and P2b (at 240 degrees)

121 (see Figure 8, 9, and 10). The third group of subjects was also presented with one of two targets

122 in each trial, but the target locations at 60 and 120 degrees from the origin were only separated by 
12360 degrees, (P3, see Figure 1B). Analogous to the previous paradigm, these targets will be

124 referred to as P3a (at 60 degrees) and P3b (at 120 degrees) (see Figure 8, 9, and 10).

125 It is important to note that since the target number differed between paradigms but the trial

126 number in each experimental block was kept constant, the number of repetitions to each target

127 differed. In the first paradigm, each target was shown 10 times in the familiarization and washout

128 blocks, and 30 times in the learning block. In the second and third paradigm, each target was

129 presented 60 times during familiarization and washout and 180 times during learning.

130 In the beginning of each trial, subjects had to position the cursor in the centre of the workspace,

131 which was indicated by a circle $1.5 \mathrm{~cm}$ in diameter. After an initial $500 \mathrm{~ms}$ waiting-period (Figure

$132 \mathrm{1C}$ ), the target (another circle $1.5 \mathrm{~cm}$ in diameter) appeared, and subjects had to reach it with the

133 cursor. At the same time, the circle showing the centre of the origin disappeared. Only after the

134 cursor was placed within the target and remained there for $50 \mathrm{~ms}$, subjects were allowed to move

135 back to the origin. This was signified by the disappearance of the target and the cursor, and the

136 reappearance of the circle representing the origin. Prior to starting the experiment, subjects were

137 instructed to move back to the centre of workspace as accurately as possible, albeit lacking visual

138 feedback about the cursor position. With regards to the speed of movements, subjects were told to

139 move at a natural pace. After they thought they had reached the origin, subjects were requested to

140 let loose of the phantom handle and put their hand on their knee until the next trial started. During

141 this period, the handle was autonomously moved back to the exact origin position by the phantom

142 device. Since subjects were not allowed to keep the phantom handle in their hand, they could not

143 feel whether or in which direction the phantom was moving to get back to the centre, and

144 therefore they had no proprioceptive information on whether or not they had actually reached the

145 origin during the backward movements. One second after the Phantom device was reset to the

146 central position, the central target and the cursor reappeared, signalling the start of the next trial

147 and instructing subjects to grasp the handle again.

148 Data Analysis

149 Before analysing the movements, we low-pass filtered the recorded trajectories (10Hz cut-off,

150 2nd order Butterworth filter). Oscillations above $10 \mathrm{~Hz}$ are unlikely to be caused by subjects'

151 movements, and are therefore assumed to represent recording artefacts. For quantification of

152 subjects' learning, we determined the error of initial movement direction. This error was defined

153 as the signed angular difference between initial movement direction and target direction from the

154 hand location at movement onset. Initial movement direction was defined as the hand position 
$155150 \mathrm{~ms}$ into the movement in relation to the hand location at movement onset. Movement onset

156 was found by a semi-automated procedure, aimed at determining a point in time when the handle

157 had not been moved from the starting location. At the same time, we wanted to exclude quivering

158 movements as well as 'false starts' from the trials entering the analysis. To this end, we defined

159 movement onset as the point in time $100 \mathrm{~ms}$ before 45 percent of maximal hand-velocity was

160 reached. In case velocity was not increasing monotonically until the threshold of 45 percent

161 maximal velocity was reached, the procedure was repeated for the next time point (and monotony

162 tested for $100 \mathrm{~ms}$ after that new time point) until the velocity increase was monotonic. After a

163 movement onset was found by the automated procedure, we visually inspected its relation to

164 both, movement trajectory and speed-profile of the movement. If either no movement onset was

165 found, or it was obviously defective, we discarded the respective trial. Initial movement direction

166 was chosen as parameter for assessing the subjects' behaviour because we wanted to assess the

167 change in the internal model of the subjects used for movement planning, before any online

168 corrections, induced by the visual feedback, took place. Although, in the literature, latencies of

169 under $150 \mathrm{~ms}$ have been described for visual feedback influencing motor control (Franklin \&

170 Wolpert, 2008), in our task, we did not observe any corrective movements before $150 \mathrm{~ms}$ after

171 movement onset (see Figure 4 for an exemplary movement, with the portion of the trajectory

172 used for determining the initial movement direction highlighted in colour).

173 To show the time course of performance and learning in the experimental blocks, we plotted

174 initial movement errors against trial number. The time course of initial movement errors was fit

175 with a linear function in the familiarization blocks, and with a single exponential function

176 according to

$177 E(t)=a+b \times e^{(-t / T)}$ in the learning and washout blocks. The parameters derived for the

178 exponential fits are shown in Table 1.

179 For each trial, we tested whether movement errors (pooled over subjects) were significantly

180 different from zero (ranksum test, $\mathrm{p}<0.01$, see Figures 5, 6, and 7). This is mostly relevant for the

181 familiarization block, in which initial movement errors from only very few trials are significantly

182 different from zero (not aimed more or less directly in the direction of the target presented).

183 For Figure 8, we pooled initial movement errors over a number of trials in the beginning and in

184 the end of the learning block to check for significant differences between forward (Figure $8 \mathrm{~A}$ )

185 and backward (Figure 8B) movements. For the statistical comparison, we used a ranksum test

186 (for the p-values, refer to figures 8 and 9). For the first paradigm, we pooled over the first and last

18750 trials, irrespective of target direction. Target direction was ignored in this paradigm because 
188 we expected a high degree of generalization of learning between nearby targets (also see

189 Discussion). For the second and third paradigm, we first sorted trials by target direction and then

190 pooled over the first and last 15 trials separately for each target (P2a and P2b in the second

191 paradigm; P3a and P3b in the third paradigm). Differences between performance in forward and

192 backward movements are shown for the beginning (Figure 8C) and the end (Figure 8D) of the

193 learning block. The same analysis for the washout block is shown in Figure 9.

194 To directly compare the learning of the rotation in forward versus backward movements between

195 the second and third paradigm, we computed the difference between movement errors in forward

196 and backward direction separately for each subject in the end of the learning block (as for Figure

1978 , we averaged over the last 15 trials), and compared these subject-wise differences between the

198 two groups (ranksum test). The individual subject-wise differences are shown in Figure 10.

\section{Results \& Discussion}

\section{Movement Trajectories}

201 Figure 2 schematically illustrates the kinds of forward and backward movements that could be expected under a visuomotor transformation, based on previous findings observed in this kind of task (Krakauer et al. 2000, Krakauer 2009 for a review on visuomotor rotations).

Figure 2A (blue arrow) shows the behaviour that can be expected for forward movements in the beginning of exposure to the transformation: Subjects would start off moving towards the visually perceived location of the target. After initiating the movement, visual feedback would make them realize that they are 60 degrees off the desired direction, leading to a large corrective movement (resulting in a hook-shaped trajectory). With prolonged exposure to the rotation, subjects would be expected to recalibrate their motor system such that they would immediately reach into the required rotated direction, producing straight trajectories again (Figure $2 \mathrm{~B}$ and $\mathrm{C}$ ).

211 Figure 2B (orange arrow) shows how subjects would move backwards if they would only take the

212 visually perceived target locations into account, failing to account for the shift induced by the

213 transformation, and making them end up in a position completely off the origin. Figure $2 \mathrm{C}$, in

214 contrast, shows how subjects would move back if they would have learned the effect of the

215 transformation, allowing them to faithfully reach the origin again. If such learning transfer to

216 backward movements was seen in all paradigms, it could be concluded that subjects learned a 
217 position remapping of all positions visited during learning in forward movements. If, however,

218 learning transfer was observed only in paradigms one and two, and not in paradigm three, it could

219 be concluded that no general position remapping took place, but rather a specific learning transfer

220 concerning the movement directions trained during forward movement. Note that backward

221 movements are expected to be always straight, since, due to the lack of visual feedback, no

222 corrective movements are expected.

223 Figure 3 shows typical real examples of movements trajectories in the three paradigms at

224 different stages of the learning block. In the forward movements of all three paradigms (Figure

$2253 \mathrm{~A}, \mathrm{C}$ and $\mathrm{E}$ ), subjects behaved as expected once the rotation was applied. In the first couple of

226 trials, there were large initial movement errors and subsequently large movement corrections to

227 reach the targets. Over the course of learning, forward movements gradually became straighter

228 and the movement direction rotated more and more to compensate for the rotation, such that the

229 target could successfully be reached. over the course of the learning block.

230 In contrast to forward movements and as expected due to the lack of feedback, backward

231 movements (Figure 3B, D, and F) were straight even in the beginning of the learning block .

232 They never displayed the typical hook-like shape induced by corrective movements. Interestingly,

233 backward movements never exactly pointed towards the origin as in Figure 3C. Rather, the initial

234 movement directions seemed to lie between the one required taking the actual hand position into

235 account and the one required based on the visually perceived hand location. In paradigms one and

236 two (Figures 3B and D), it seemed that the backward movement direction would shift over the

237 course of the learning block towards the direction required by the visuomotor rotation.

238 Initial Movement Errors

239 To quantify motor behaviour and check for systematic changes in movement direction over the

240 course of the experiment, we computed initial errors in forward and backward movement

241 directions and plotted them against time. For better visualization and quantification of the results,

242 we fitted the forward and backward movement errors of the training and washout blocks with an

243 exponential function (see methods for procedure and Table 1 for the estimated parameters of the

244 fits).

245 For the presentation of initial movement errors in the first paradigm, we ignored target directions

246 when looking at the time-course of learning (see Figure 5). Due to the pseudo-random

247 presentation of many target directions, consecutive trials to the same target can be separated by

248 many trials to other targets. If targets are learned completely separately, this should not strongly 
249 affect the time-course of learning the rotation for single targets (see Krakauer et al. 2000), but in

250 our first paradigm, targets were quite close together (30 degree) so we expected to see at least

251 some generalization between nearby target locations. Instead of trying to correct for this during

252 the analysis (e.g. by taking into account the presentation order of targets), we decided to look at

253 consecutive trials irrespective of target direction for the paradigm with 12 targets.

254 In the second and third paradigm, target locations were separated by at least 60 degrees (in

255 paradigm three), so we did expect much less, if any, generalization across target directions. In the

256 last two paradigms, we therefore separated trials by target direction and looked at the time-course

257 of learning separately for each of them. This also enabled us to compare movements to the same

258 location in the workspace between paradigms: the location of target P2a in the second paradigm

259 is identical to that of target P3a in the third paradigm (see Figure 6 and 7). The different numbers

260 of targets presented in the paradigms mean that one has to be cautious in comparing learning

261 between the first and the last two paradigms. The conclusions drawn in the following are

262 therefore most critically based on the comparisons of forward and backward movements within a

263 given paradigm and the comparison of paradigm 2 and 3 (in which equal numbers of repetitions

264 were used). Therefore, the factor of target numbers is not confounding our results.

265 Forward Movements

266 In forward movements (blue dots and lines in Figures 5, 6, and 7), as expected, when the rotation

267 of the visual feedback was switched on, subjects in all groups started off with initial movement

268 errors close to the magnitude of the rotation (60 degrees, see Figures 5, 6, and 7, blue dots). In the

269 following trials, initial movement errors decreased until reaching a plateau at which performance

270 remained relatively constant.

271 Performance in the washout block (right panel of Figures 5, 6, and 7) confirmed that a typical

272 sensorimotor learning process had taken place in forward movements. In all experiments, a

273 distinct after-effect was observed: in the beginning of the washout block subjects started off with

274 large errors in the direction opposite to the transformation. These errors decreased rapidly over

275 the following trials.

276 In accordance with the learning process, over the course of both the learning and the washout

277 block, the occurrence of significant differences from zero (ranksum test, based on the movements

278 of all subjects in each experimental group for individual trials), seemed to consistently decrease

279 (blue lines in the bottom of Figures 5, 6, and 7). Given the low statistical power of comparing a

280 sample of only 10 to a mean of zero, these data have to be interpreted with caution, however. 
281 For the comparison of initial movement errors in the beginning and in the end of the learning

282 block, we pooled over the first and last 50 trials of the block for paradigm one, and over the first

283 and last 15 trials for paradigms two and three. Pooling over a large number of trials will give

284 more reliable results given the variability of initial movement errors, but it will fail to capture the

285 initial phase of the experiment in which the movements are not learned, yet. In the first paradigm,

286 taking 50 trials was reasonable looking at the time-course of learning over consecutive trials

287 (Figure 5). In the second and third paradigm, especially when looking at targets separately

288 (Figure 6 and 7), learning is faster, so we chose a smaller number of trials to represent the

289 beginning and the end of learning respectively. Comparing the pooled trials from the beginning

290 and the end of the learning block yielded a highly significant drop of errors in all experiments,

291 showing that substantial learning was achieved in forward movements (Figure 8A). Note that this

292 finding is obvious to some degree, since subjects who did not show a significant decrease in

293 forward movement errors were not included in the analysis. In the washout block, we found

294 substantial unlearning, again indicated by a significant drop in the initial movement errors from

295 the first to the last trials in the block (Figure 9A).

296 Subjects' movement errors in the third paradigm generally seemed to be smaller (both in the

297 beginning and in the end of the learning block) than in the second paradigm (see Figure 8A). This

298 could, for instance, reflect some generalization of learning between targets (targets were only 60

299 degrees apart in the third, but 180 degrees apart from each other in the second paradigm),

300 however, further experiments would be needed to investigate this effect in detail.

301 The learning and unlearning processes seemed to be well captured by the exponential fits (see

302 Table 1 for the parameters of the exponential fits).

\section{Backward movements}

304 In backward movements (orange dots and lines in Figures 5, 6, and 7), the initial movement

305 errors in the very first trials of the learning block were typically smaller than those observed in

306 forward movements (see Figure 8C). This is in agreement with the observation in movement

307 trajectories that subjects seemed to plan movements in directions between those required on the

308 basis of the visual and on the basis of the proprioceptive information about the hand's location.

309 Apparently, backward movements were planned by integrating proprioceptive information about

310 the actual hand starting position and the visual information about the cursor position, which is in

311 agreement with other psychophysical experiments (van Beers, Sittig and Denier van der Gon,

312 1996; van Beers, Sittig and Denier van der Gon, 1999). 
313 In addition, in backward movements, as in forward movements, errors decreased over the course

314 of the learning and the washout blocks, indicating that the transformation was also learned in

315 backward movements. Since subjects did not get sensory feedback about their performance in

316 backward movements, a learning mechanism depending on the observation of movement errors,

317 like in forward movements, was not possible.

318 Instead, subjects may have learned over the course of the learning block to change the relative

319 weight they attribute to proprioception as compared to vision when planning their movements. It

320 has been shown that the integration of visual and proprioceptive information used for movement

321 control can be altered by task circumstances (Touzalin-Chretien, Ehrler \& Dufour, 2010).

322 Generally, subjects tend to rely more heavily on vision than on proprioception for the planning

323 and execution of movements (e.g. Botvinick \& Cohen, 1998; Ernst \& Banks, 2002) and

324 proprioceptive information is even suppressed in the beginning of reaching movements (Shapiro,

325 Gottlieb \& Corcos, 2004; Shapiro et al. 2009; Niu, Corcos \& Shapiro, 2012). During the learning

326 block, subject might learn that the visual feedback they get is unreliable, and chose to disregard it

327 more and more for the planning of the backward movements. Indeed, depending on availability

328 and/or reliability of sensory information, the weighting of proprioceptive and visual information

329 has been found to be subject to change (Botvinick \& Cohen, 1998; van Beers, Sittig \& Denier

330 van der Gon, 1999; Ernst \& Banks, 2002; Sober \& Sabes, 2003).

331 Alternatively subjects might learn the shifted target locations during forward movements

332 (consistent with the position remapping hypothesis), and switch to location remapping for

333 planning their backward movements.

334 Both these learning mechanisms would rely on subjects being able to adopt more than one

335 learning strategy, and switch between them. Indeed, subjects have been reported to readily learn

336 more than one transformation in case they are training in different contexts (Thomas \& Bock,

337 2012), and to be able to switch between different control mechanisms depending on that context

338 (Scheidt \& Ghez, 2007; Ghez, Scheidt \& Heijing, 2007; Scheidt, Ghez \& Asnani, 2011). The

339 absence of visual feedback in backward movements could function as a cue to switch context,

340 allowing them either to reweigh the available proprioceptive and visual information or to switch

341 from the control of movement direction when visual feedback is provided to a location

342 remapping mode when it is absent. Very recently, there has been a study proposing that both, the

343 rotated goal location and the rotated direction of movements are learned when subjects are

344 confronted with a visuomotor rotation. This study is in accordance with our results, and also

345 includes a computational model for the learning mechanisms. However, this study - like the ones 
346 before - tested the directions in a separate location of the workspace (Wu \& Smith, 2013).

347 In our experiment, it was not possible to determine which of the above described mechanisms

348 accounted for the observed learning, or whether maybe both were involved.

349 Moreover, there is a third mechanism, that we think plays a major role in the learning of the

350 backward movements - the direction-specific transfer of learning from forward to backward

351 movements. In the end of the learning block, performance during backward movements in the

352 first two paradigms almost reached the level observed in forward movements, while in the third

353 paradigm, it was significantly lower (Figure 8D). Actually, the residual error in the end of

354 learning in paradigm 3 was more than twice as large in backward movements as compared to

355 forward movements.

356 The difference between the last two paradigms was most striking when comparing the movement

357 errors to the target located at 60 degrees - target $\mathrm{P} 2 \mathrm{a}$ in the second, and $\mathrm{P} 3 \mathrm{a}$ in the third paradigm.

358 The location of the respective target is the same in both groups (making this comparison as fair as

359 it can get), and the number of repetitions to the target, as well as the number of additional targets

360 presented (one) are the same in the last two paradigms. And yet, in P2a, there was only a slight

361 difference in the movement errors between forward and backward movements in the end of the

362 learning block, whereas in P3a the difference was large and highly significant (Figures 8D, P2a

363 and P3a, ranksum test, p-values see figures). When doing a subject-wise comparison of the

364 difference between forward and backward movement errors between paradigms (see methods for

365 details), we found that the difference was indeed significantly larger in the third paradigm

366 (ranksum test, $\mathrm{p}<0.05$ ), both when looking only at target $\mathrm{P} 2 \mathrm{a} / \mathrm{P} 3 \mathrm{a}$ as well as when pooling over

367 all three targets presented in the two paradigms. We propose that this difference is caused by a

368 direction-specific transfer of learning from forward to backward movements. The differences

369 between forward and backward movement errors for single subjects (Figure 10) reveal that,

370 indeed, the difference between forward and backward movement errors was generally larger in

371 the paradigm in which targets were not located opposite each other. Additionally, this figure

372 shows that there is one subject in paradigm three who acts contrary to any other subject in the

373 group (the last bar on the right in both Figure 10A and B). There was no objective reason to

374 exclude this subject from the analysis, however.

375 Direction-specific transfer of learning has been described before (Krakauer et al. 2000, Sainburg

376 et al. 2003), and, in addition, it was shown that learning of visuomotor rotations was transferred

377 between movements starting at different locations of the workspace, as long as they were in the

378 same direction (Wang \& Sainburg, 2005). In contrast to these earlier studies, however, our study 
379 has the advantage that the learning transfer was tested in an area of the workspace that was

380 completely overlapping with the area trained during learning. Therefore, the workspace could be

381 excluded as a confounding variable against location remapping. Our results are consistent with

382 the view that movement direction is a major parameter specified during motor programming,

383 encoded separately from position in the motor system, as postulated by the vectorial planning

384 hypothesis. The idea that movements are primarily planned on the basis of movement direction is

385 also supported by neurophysiological findings that movement direction is a prominent parameter

386 encoded in neuronal activity (Georgopoulos et al., 1986). On the basis of this assumption,

387 planned movements can amazingly accurately and robustly be decoded from neural signals in

388 monkeys (Schwartz 1994). Directional coding of movements seems also to occur in humans

389 (Cowper-Smith et al. 2010, Fabbri et al. 2010). Brain-machine interfaces can quite successfully

390 exploit decoding of intended movement directions for steering machines or prostheses with brain

391 signals, both in monkeys (Taylor, Tillery \& Schwartz 2002) as well as in humans (Milekovic et

392 al. 2012, Hochberg et al. 2012).

393 We propose that the three mechanisms discussed above - the re-weighting of sensory

394 information, the learning of transformed target location, and the (direction-specific) transfer of

395 learning from forward movements - are the most important factors leading to the adaptation to

396 the rotation of movement directions seen in backward movements. However, there are two

397 further possibilities that we want to discuss: First, Learning might at least partly have been

398 caused by non-specific generalization of learning (generalization across directions). Although

399 Krakauer et al. in their original study claim that the learning of a visuomotor rotation is local with

400 regards to the direction of movements, their results suggest at least some degree of generalization

401 (Krakauer et al., 2000, Figures 7A and B), especially where there is more than one training

402 direction (see also Brayanov, Press \& Smith, 2012). We think, however, that at 120 degrees, the

403 difference between forward and backward movement directions in paradigm three was large

404 enough to render generalization across them unlikely.

405 Secondly, subjects might have made use of the mismatch between the location where they left the

406 handle of the tracking device in the end of each trial and the position in which they took hold of it

407 again after the Phantom had moved the handle towards the origin, and started the new trial. To

408 minimize this kind of information, subjects were told to put their hands well away from the

409 tracking device and put them on their lap between trials. Since the movements bringing back the

410 Phantom to the origin were quite small, and given this intermittent movement to the subjects' lap

411 and back, we think this effect is negligible. 
412 While we therefore are confident that we can exclude the latter explanations for the learning in

413 backward movements, further experiments would be necessary to distinguish between the other

414 two explanations mentioned and specify the magnitude to which each adds to the observed

415 learning effect.

416 To summarize, the main two findings of our study, shedding light on the planning of backward

417 movements in human subjects are: First, when confronted with a transformation at a given

418 moment, the following movement (in our case, the backward movement) is planned based on a

419 mixture of visual and proprioceptive information, suggesting that the transformation induces a re-

420 weighting of information coming from the two perceptual channels. This has been shown before

421 for other types of movements, but to our knowledge, it is a new finding when looking at

422 backward movements specifically. We think that this is relevant because backward movements

423 are an integral part of reaching movements, and it would therefore not be implausible to assume

424 that they are planned along with the forward movements, integrated in one motor command. Our

425 results suggest in contrast, that backward movements are planned separately from the preceding

426 forward movements.

427 Secondly, we have shown that the learning of a visuomotor rotation in forward directions also

428 induces learning in the following backward movements of out-and-back reaching movements.

429 There are several mechanisms which could be relevant in this respect, and further experiments

430 would be needed to determine their specific contributions to the observed learning effect, but our

431 results strongly suggest that one of them is a direction-specific transfer of learning from forward

432 to backward movements, supporting the vectorial planning hypothesis of motor control and

433 emphasizing the role of direction as an important control parameter within the motor system.

\section{Acknowledgements}

435 The authors would like to thank Carsten Mehring (University of Freiburg) for long-term support

436 and advice and Dmitry Kobak (Champalimaud Neuroscience Programme) for helpful

437 discussions. 
439 van Beers RJ, Sittig AC, Denier van der Gon JJ. 1996. How humans combine simultaneous

440 proprioceptive and visual position information. Experimental Brain Research 111(2):253-61.

441 van Beers RJ, Sittig AC, Denier van der Gon JJ. 1999. Integration of proprioceptive and visual 442 position-information: An experimentally supported model. Journal of Neurophysiology 81:135544364.

444 Botvinick M, Cohen J. 1998. Rubber hands 'feel' touch that eyes see. Nature 391:756.

445 Brayanov JB, Press DZ, Smith MA. 2012. Motor memory is encoded as a gain-field combination 446 of intrinsic and extrinsic action representations. The Journal of Neuroscience 32:14951-14965.

447 Cowper-Smith CD, Lau EY, Helmick CA, Eskes GA, Westwood DA. 2010. Neural coding of 448 movement direction in the healthy human brain. PLoS One 13;5(10):e13330.

449 Ernst MO, Banks MS. 2002. Humans integrate visual and haptic information in a statistically 450 optimal fashion. Nature 415:429-33.

451 Fabbri S, Caramazza A, Lingnau AJ. 2010. Tuning curves for movement direction in the human 452 visuomotor system. The Journal of Neuroscience. 6;30(40):13488-98.

453 Franklin DW, Wolpert DM. 2008. Specificity of reflex adaptation for task-relevant variability. 454 The Journal of Neuroscience 28:14165-75.

455 Georgopoulos AP, Schwartz AB, Kettner RE. 1986. Neuronal population coding of movement 456 direction. Science 26;233(4771):1416-9.

457 Ghez C, Scheidt R, Heijing H. 2007. Different learned coordinate frames for planning trajectories 458 and final positions in reaching. Journal of Neurophysiology 98:3614-3626.

459 Gordon J, Ghilardi MF, Ghez C. 1994. Accuracy of planar reaching movements. I. Independence 460 of direction and extent variability. Experimental Brain Research 99:97-111. 
461 Hochberg LR, Bacher D, Jarosiewicz B, Masse NY, Simeral JD, Vogel J, Haddadin S, Liu J, Cash

462 SS, van der Smagt P, Donoghue JP. 2012. Reach and grasp by people with tetraplegia using a

463 neurally controlled robotic arm. Nature. 16;485(7398):372-5.

464 Imamizu H, Uno Y, Kawato M. 1995. Internal Representations of the Motor Apparatus:

465 Implications From Generalization in Visuomotor Learning. Journal of Experimental Psychology:

466 Human Perception and Performance 21(5):1174-1198.

467 Krakauer JW. 2009. Motor Learning and Consolidation: The Case of Visuomotor Rotation.

468 Advances in Experimental Medicine and Biology 629:405-421.

469 Krakauer JW, Pine ZM, Ghilardi MF, Ghez C. 2000. Learning of visuomotor transformations for 470 vectorial planning of reaching trajectories. The Journal of Neuroscience 20:8916-24.

471 Messier J, Kalaska JF. 1999. Comparison of variability of initial kinematics and endpoints of 472 reaching movements. Experimental Brain Research 125:139-52.

473 Milekovic T, Fischer J, Pistohl T, Ruescher J, Schulze-Bonhage A, Aertsen A, Rickert J, Ball T, 474 Mehring C. 2012. An online brain-machine interface using decoding of movement direction from 475 the human electrocorticogram. Journal of Neural Engineering 9(4):046003.

476 Niu CM, Corcos DM, Shapiro MB. 2012. Suppression of proprioceptive feedback control in 477 movement sequences through intermediate targets. Experimental Brain Research 216:191-201.

478 Oldfield RC. 1971. The assessment and analysis of handedness: the Edinburgh inventory. 479 Neuropsychologia 9(1):97-113.

480 Polit A, Bizzi E. 1978. Processes controlling arm movement in monkeys. Science 201:1235-1237.

481 Rosenbaum DA, Loukopoulos LD, Meulenbroek RG, Vaughan J, Engelbrecht SE. 1995. Planning 482 reaches by evaluating stored postures. Psychological Review 102:28-67 
483 Rosenbaum DA, Meulenbroek RJ, Vaughan J. 1999. Remembered positions: stored locations or 484 stored postures? Experimental Brain Research 124:503-512.

485 Sainburg RL, Lateiner JE, Latash ML, Bagesteiro LB. 2003. Effects of altering initial position on 486 movement direction and extent. Journal of Neurophysiology 89:401-15.

487 Scheidt RA, Ghez C. 2007. Separate adaptive mechanisms for controlling trajectory and final 488 position in reaching. Journal of Neurophysiology 98:3600-3613.

489 Scheidt RA, Ghez C, Asnani S. 2011. Patterns of hypermetria and terminal cocontraction curing 490 point-to-point movements demonstrate independent action of trajectory and postural controllers. 491 Journal of Neurophysiology 106:2368-2382.

492 Schwartz AB. 1994. Direct cortical representation of drawing. Science 22;265(5171):540-2.

493 Shapiro MB, Gottlieb GL, Corcos DM. 2004. EMG responses to an unexpected load in fast 494 movements are delayed with an increase in the expected movement time. Journal of 495 Neurophysiology 91:2135-2147.

496 Shapiro MB, Niu CM, Poon C, David FJ, Corcos DM. 2009. Proprioceptive feedback during 497 point-to-point arm movements is tuned to the expected dynamics of the task. Experimental Brain 498 Research 195:575-591.

499 Sober SJ, Sabes PN. 2003. Multisensory integration during motor planning. The Journal of 500 Neuroscience 23:6982-92.

501 Thomas M, Bock O. 2012. Concurrent adaptation to four different visual rotations. Experimental 502 Brain Research 221:85-91.

503 Touzalin-Chretien P, Ehrler S, Dufour A. 2010. Dominance of Vision over Proprioception on 504 Motor Programming: Evidence from ERP. Cerebral Cortex 20:2007-2016. 
505 Vindras P, Desmurget M, Prablanc C, Viviani P. 1998. Pointing errors reflect biases in the 506 perception of the initial hand position. Journal of Neurophysiology 79:3290-4.

507 Wang J, Sainburg RL. 2005. Adaptation to visuomotor rotations remaps movement vectors, not 508 final positions. The Journal of Neuroscience 25:4024-30.

509 Wu HG, Smith MA. 2013. The generalization of visuomotor learning to untrained movements

510 and movement sequences based on movement vector and goal location remapping. The Journal 511 of Neuroscience 26;33(26):10772-89. 


\section{Table 1 (on next page)}

Parameters of curves fit to initial movement errors

P1 - Paradigm 1, all 12 Targets. P2a - Paradigm 2, target at $60^{\circ}$. P2b - Paradigm 2, target at $240^{\circ}$. P3a - Paradigm 3, target at $60^{\circ}, \mathrm{P} 3 \mathrm{~b}-$ Paradigm 3, target at $120^{\circ}$. For each parameter of the fit, the confidence intervals are given, MSE - mean squared error. 
Table1. Parameters of curves fit to Initial Movement Errors

\begin{tabular}{|l|l|l|l|l|l|l|}
\hline Paradigm & Figure & target & $\mathrm{a}$ & $\mathrm{b}$ & $\tau$ & $\mathrm{MSE}$ \\
\hline P1 forward & 3 & all & $8.3-11.9$ & $33.4-38.5$ & $81.5-120.3$ & 276 \\
\hline P1 backward & 3 & all & $12.9-14.4$ & $17.8-30.0$ & $15.4-31.8$ & 281 \\
\hline P2a forward & 4 A & $60^{\circ}$ & $15.5-18.2$ & $30.2-38.9$ & $23.9-38.1$ & 262 \\
\hline P2a backward & 4A & $60^{\circ}$ & $18.4-20.2$ & $18.9-36.1$ & $5.3-13.2$ & 257 \\
\hline P2b forward & 4 B & $240^{\circ}$ & $14.2-17.9$ & $29.2-35.6$ & $34.5-56.6$ & 216 \\
\hline P2b backward & 4 B & $240^{\circ}$ & $15.4-17.5$ & $10.6-52.2$ & $0.8-6.7$ & 397 \\
\hline P3a forward & 5 A & $60^{\circ}$ & $8.8-10.8$ & $36.2-45.4$ & $19.1-27.3$ & 207 \\
\hline P3a backward & 5 A & $60^{\circ}$ & $23.5-26.3$ & $7.6-18.4$ & $8.2-46.6$ & 350 \\
\hline P3b forward & 5 B & $120^{\circ}$ & $7.7-9.6$ & $21.5-29.3$ & $18.1-30.5$ & 179 \\
\hline P3b backward & 5 B & $120^{\circ}$ & $14.5-15.8$ & $-3.3-15.5$ & $-5.3-16.3$ & 156 \\
\hline
\end{tabular}




\section{Figure 1}

\section{Experimental Setup}

(A) Subject sitting in front of the setup. (B) Target locations in the three paradigms (unfilled circle represents the starting location). (C) Trial sequence. The phantom device was programmed to autonomously move back to the location of the origin during the last $2000 \mathrm{~ms}$ while subjects were instructed to release the handle and wait for reappearance of the origin. 
A

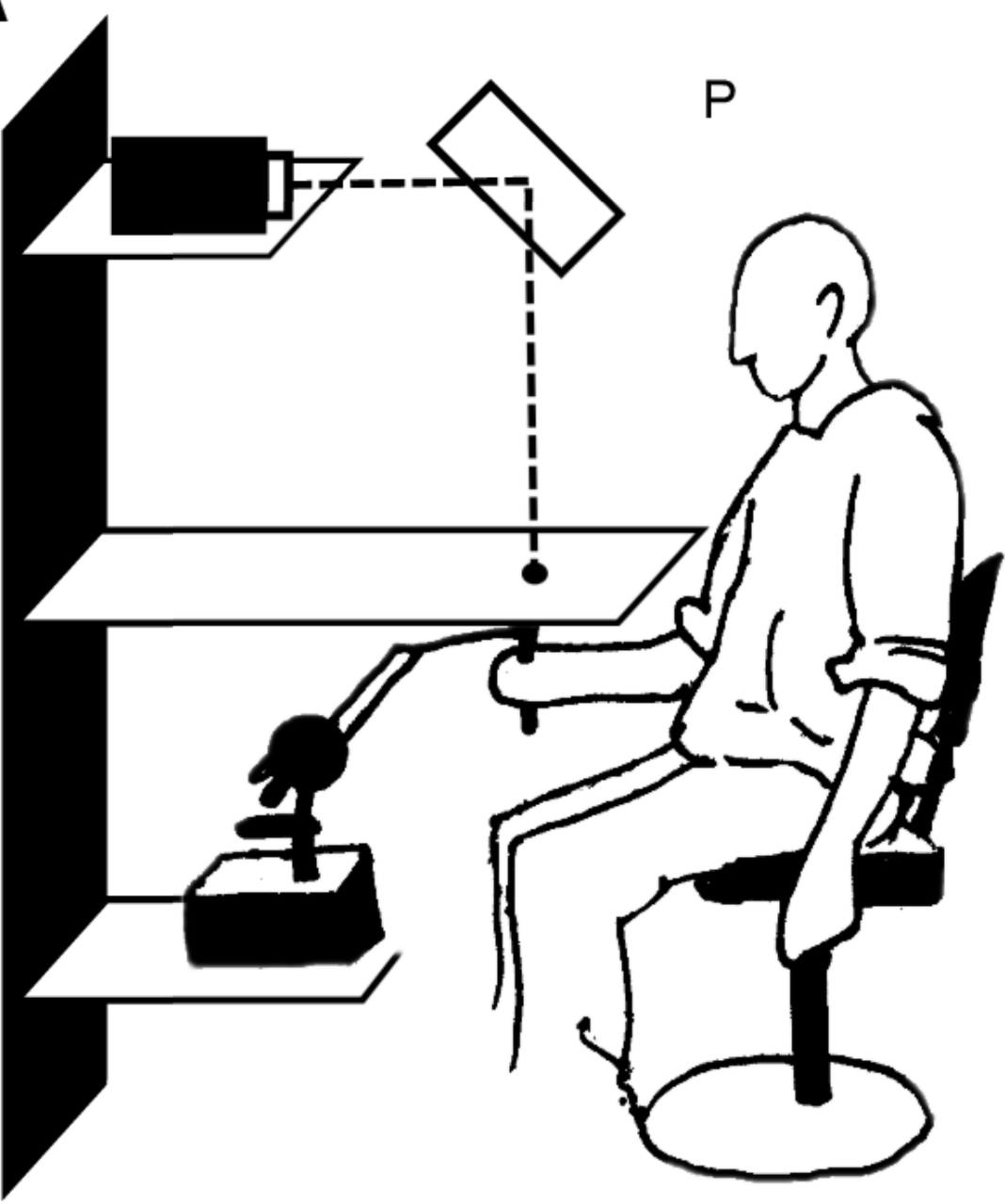

B

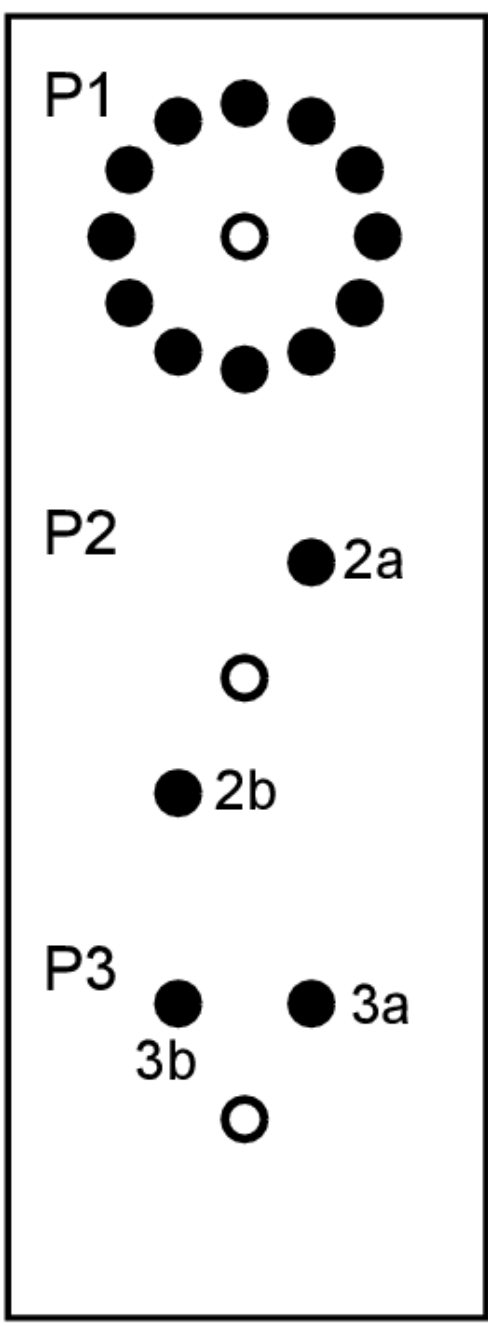

C

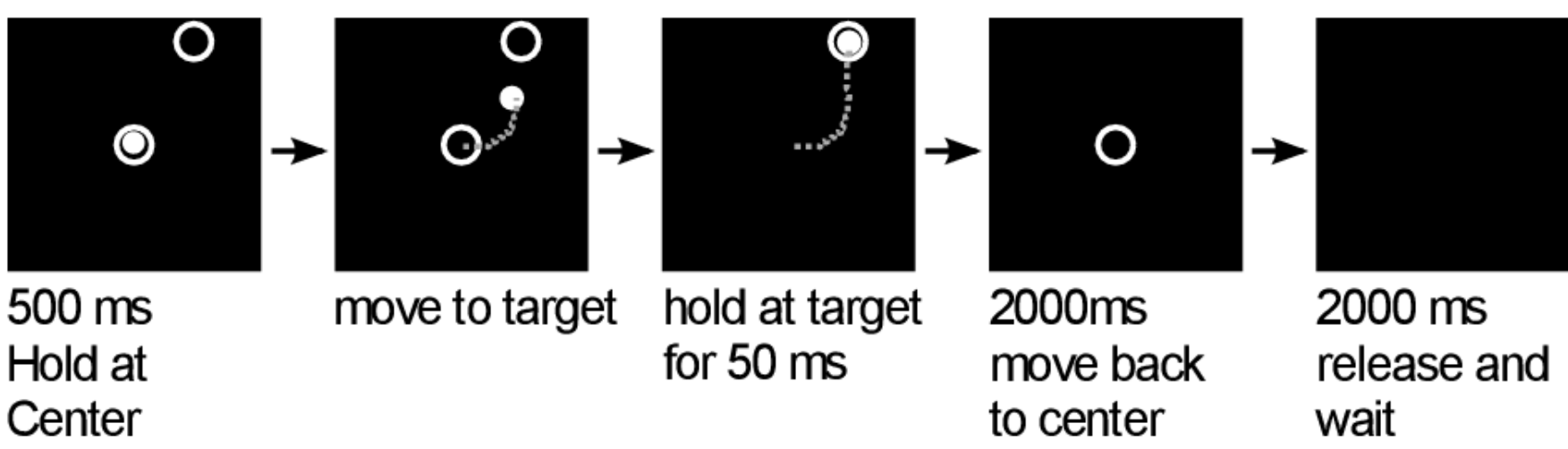




\section{Figure 2}

Expected Trajectories in our Task

(A) Initial forward movement under 60 degree clockwise (CW) visuomotor rotation (beginning of training). (B) Expected forward movement after learning the transformation and expected backward movement based on visual information of target position. (C) Expected forward movement after learning the transformation and expected backward movement, based on hand position.

A

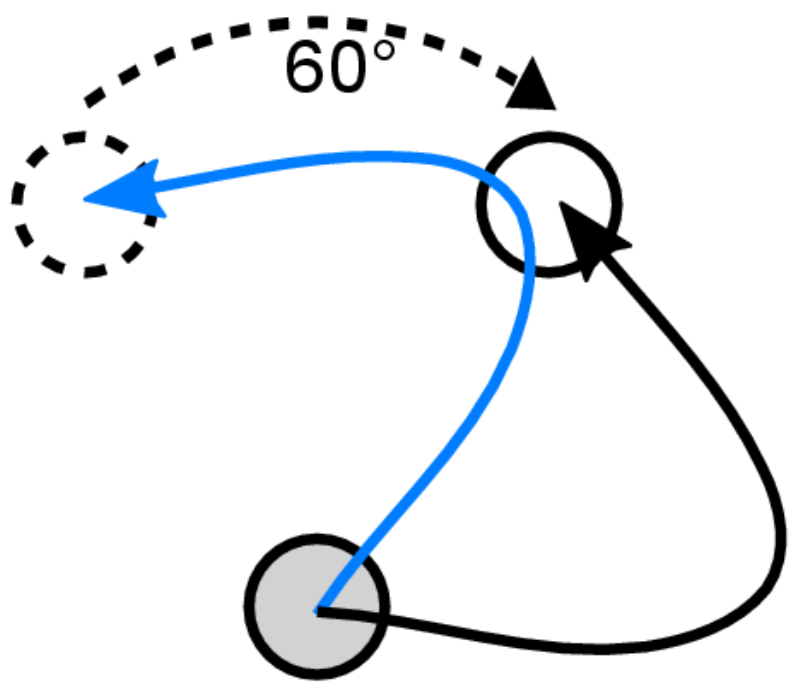

- Cursor-Feedback

- Forward Hand-Movement

_ Backward Hand-Movement

O Movement Start

V Visual Target

$\therefore$ Hand Location
B

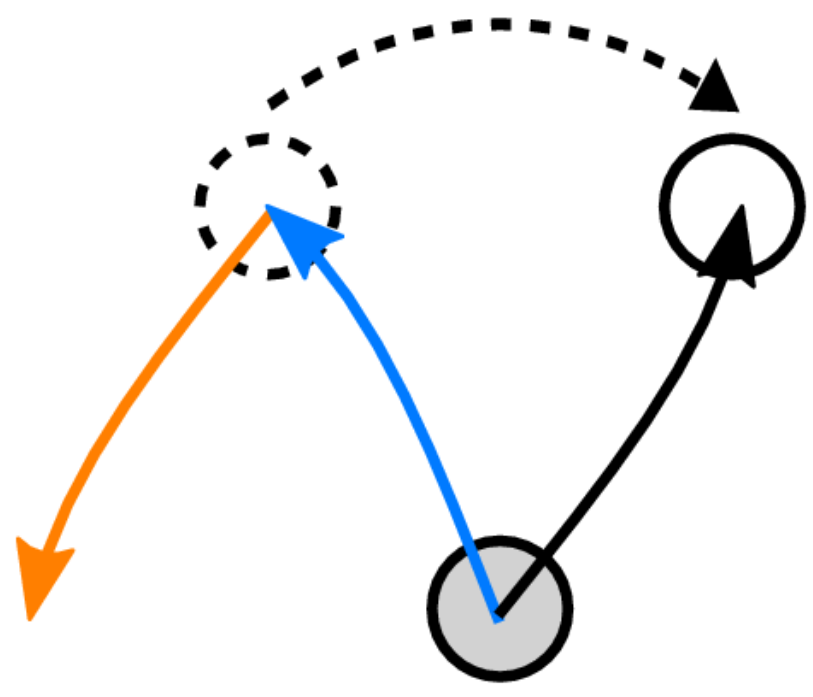

C

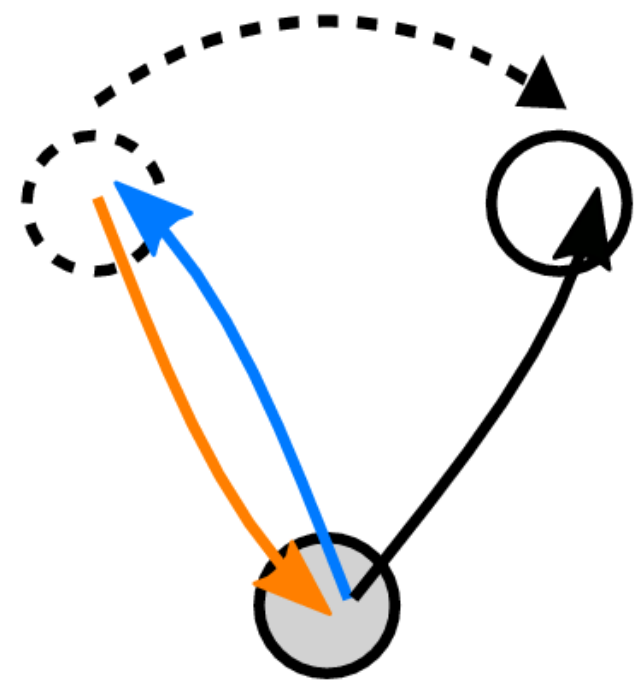




\section{Figure 3}

\section{Exemplary Trajectories During Different Stages of Learning.}

Trajectories are shown for one representative subject from each of the three experimental groups. Dotted lines denote the first successful trial, dashed lines a trial during learning (the 8th trial) and the solid lines one of the last successful trials in the block (the 17th in the first experiment, the 173rd for the second and third). (A) Forward movements in paradigm 1. (B) Backward movements in paradigm 1. (C) Forward movements in paradigm 2. (D) Backward movements in paradigm 2. (E) Forward movements in paradigm 3. (F) Backward movements in paradigm 3. The red and blue dots show at what moment initial movement errors were detected in the respective trials (see methods). Locations of the rotated targets are given by the dashed target-circles. All trajectories are shown in hand-space. 

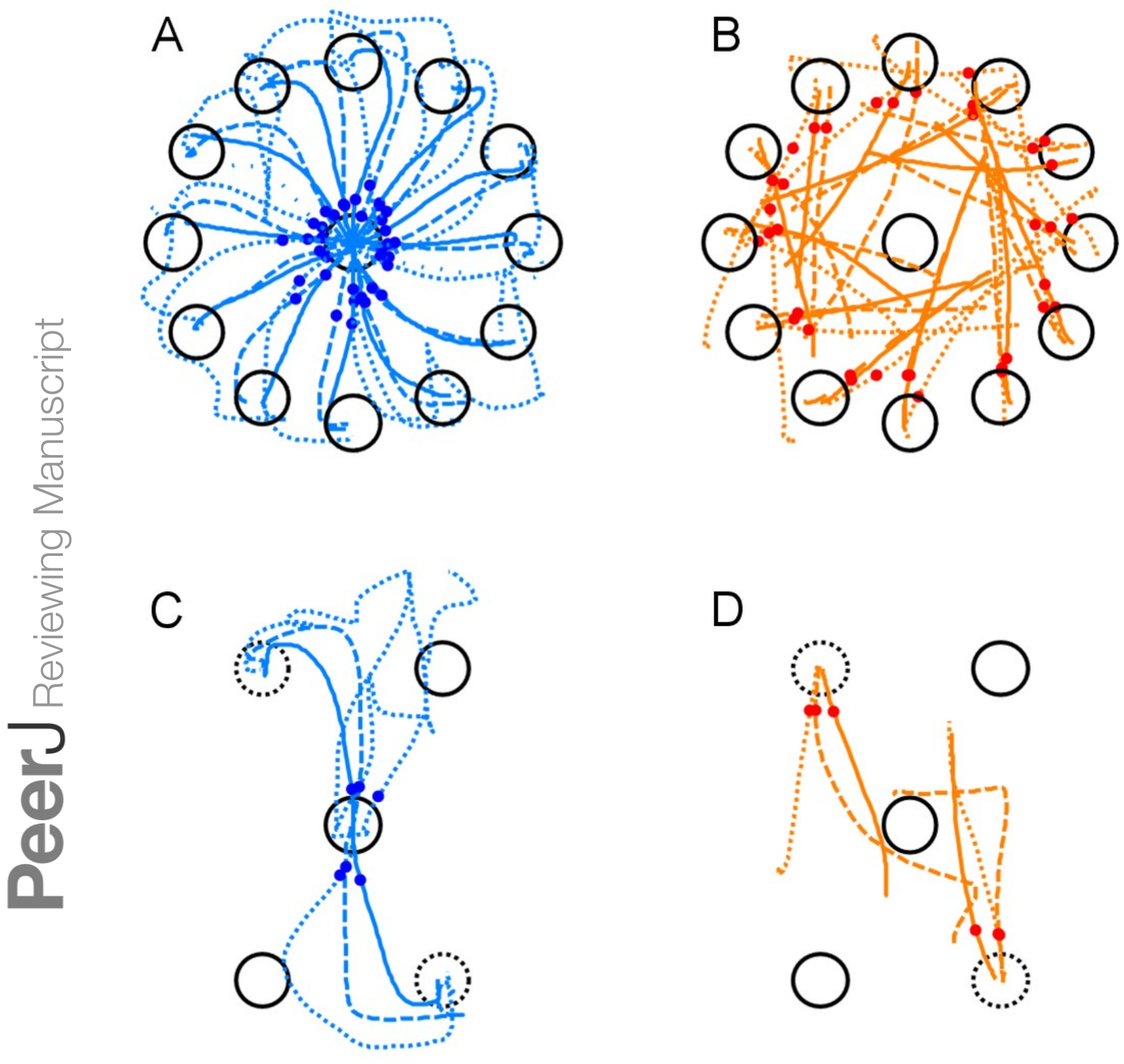

D
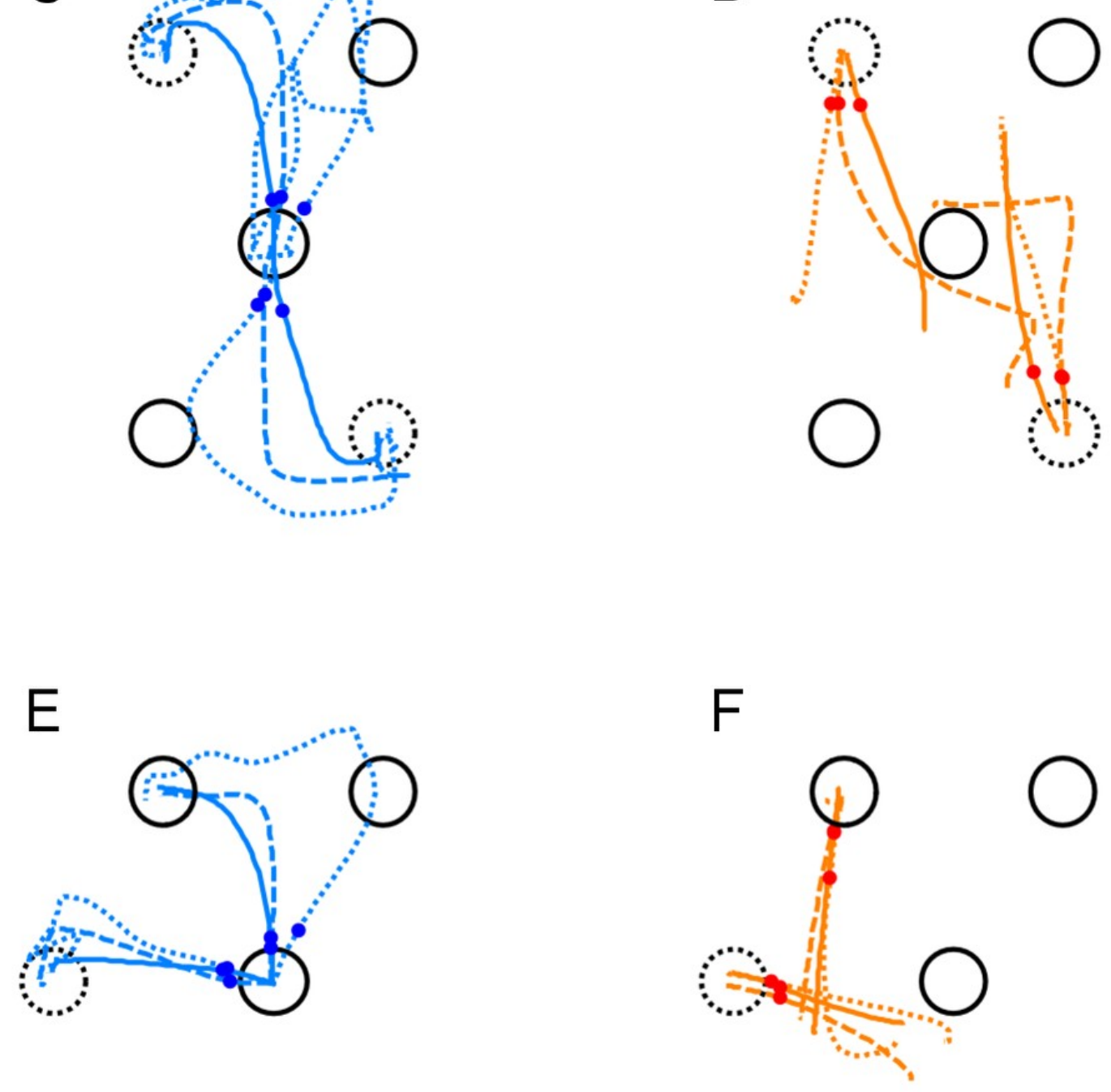

Forward Movements

Backward Movements 


\section{Figure 4}

Detection of Initial Movement Error.

The coloured parts of the trajectories show the interval of the movement which was used to define the initial movement error (in blue for the forward movement, in orange for the backward movement). Note that the corrective movement in forward direction starts well after the point in time when initial movement error was detected.

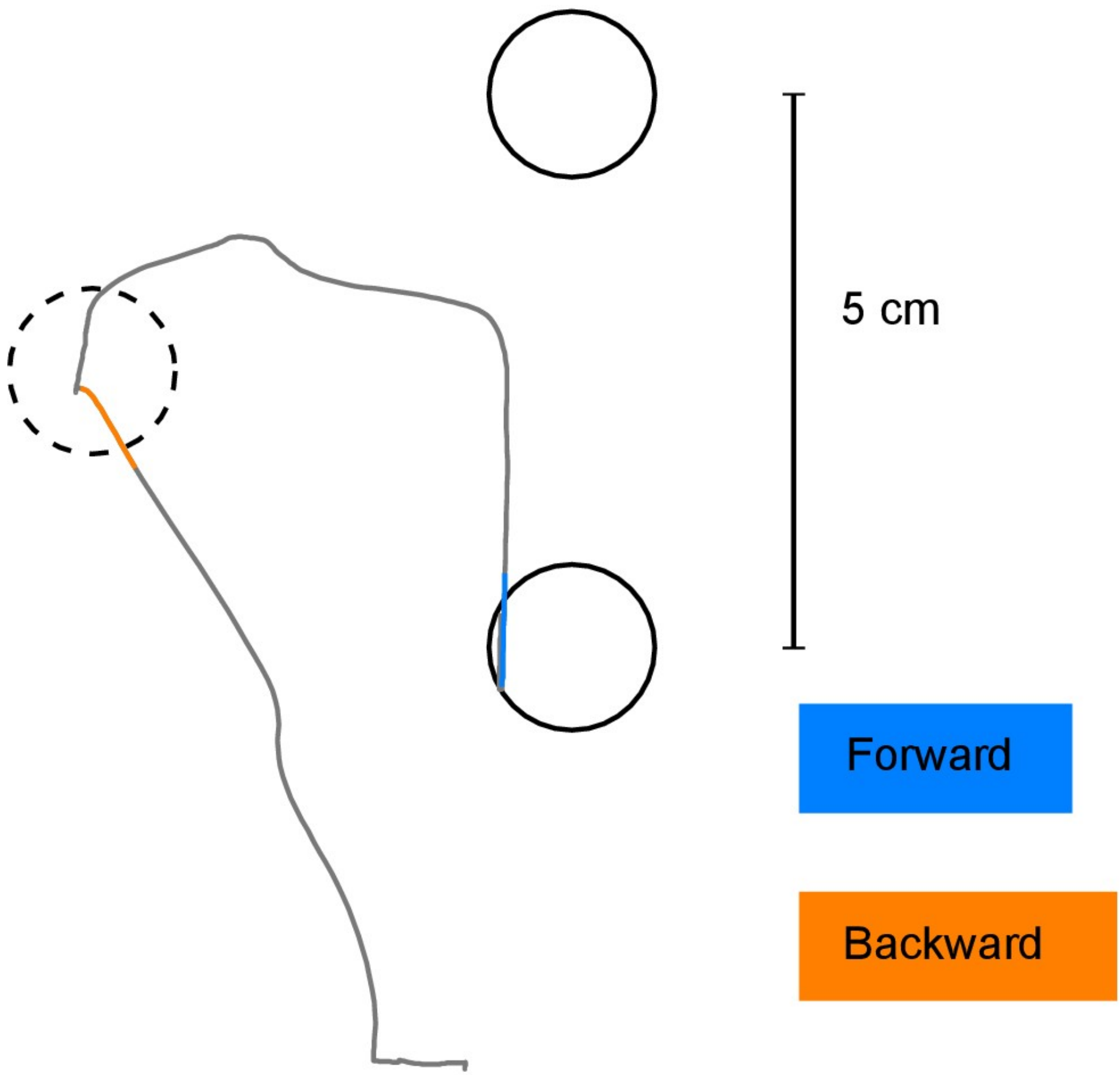




\section{Figure 5}

Initial Movement Errors (IME) in the First Paradigm (P1) with 12 Targets.

In the familiarization block (far left) and the washout block (far right), feedback was veridical. In the learning block (middle), visual feedback was rotated around the movement origin by 60 degree (CW). Individual errors are shown as dots, solid lines denote the exponential fits to the data (for confidence intervals of the fit parameters, refer to Table 1). The bars at the bottom off the graphs denote individual trials in which the errors were significantly different from zero (ranksum test, $p<0.01$ ). Forward movements are shown in blue, backward movements in orange.

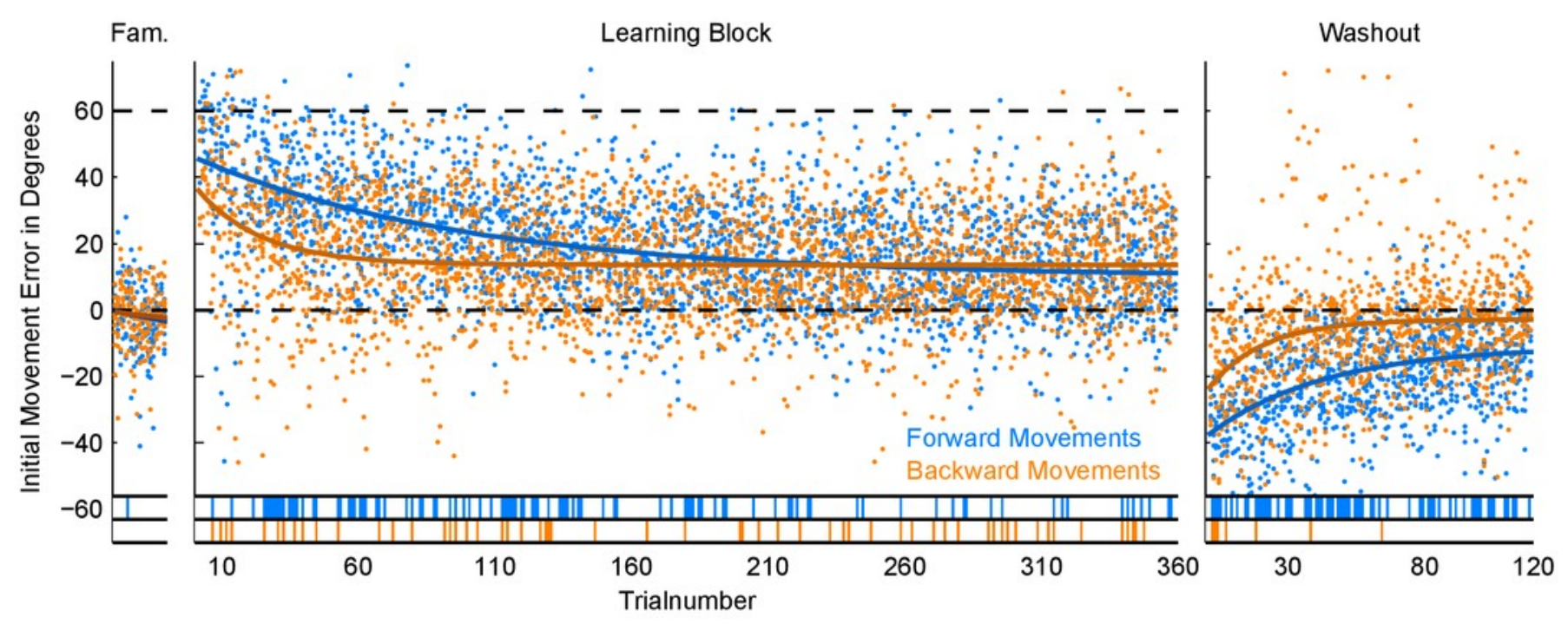




\section{Figure 6}

Initial Movement Errors (IME) in Paradigm 2 (P2 - targets located 180 degree apart).

(A) Results for the trials to the target at 60 degree. (B) Results for the target at $240^{\circ}$ in the bottom row of the figure. Refer to Figure 3 for further details.
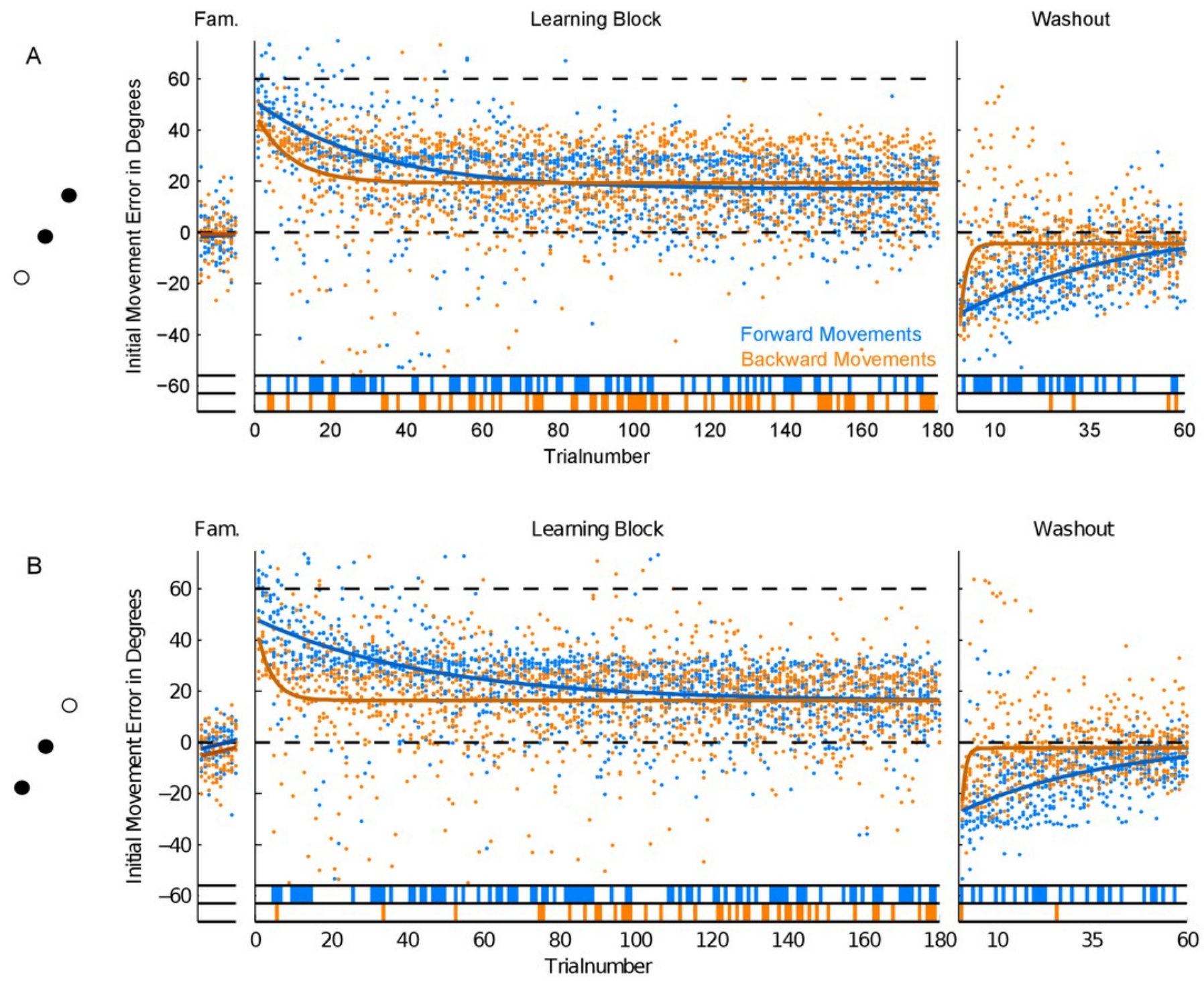


\section{Figure 7}

Initial Movement Errors (IME) in Paradigm 3 (P3 - targets located 60 degree apart).

Results for the trials to the target at 60 degree are shown in the top row, results for the target at 120 degree in the bottom row of the figure. Refer to Figure 3 for further details.
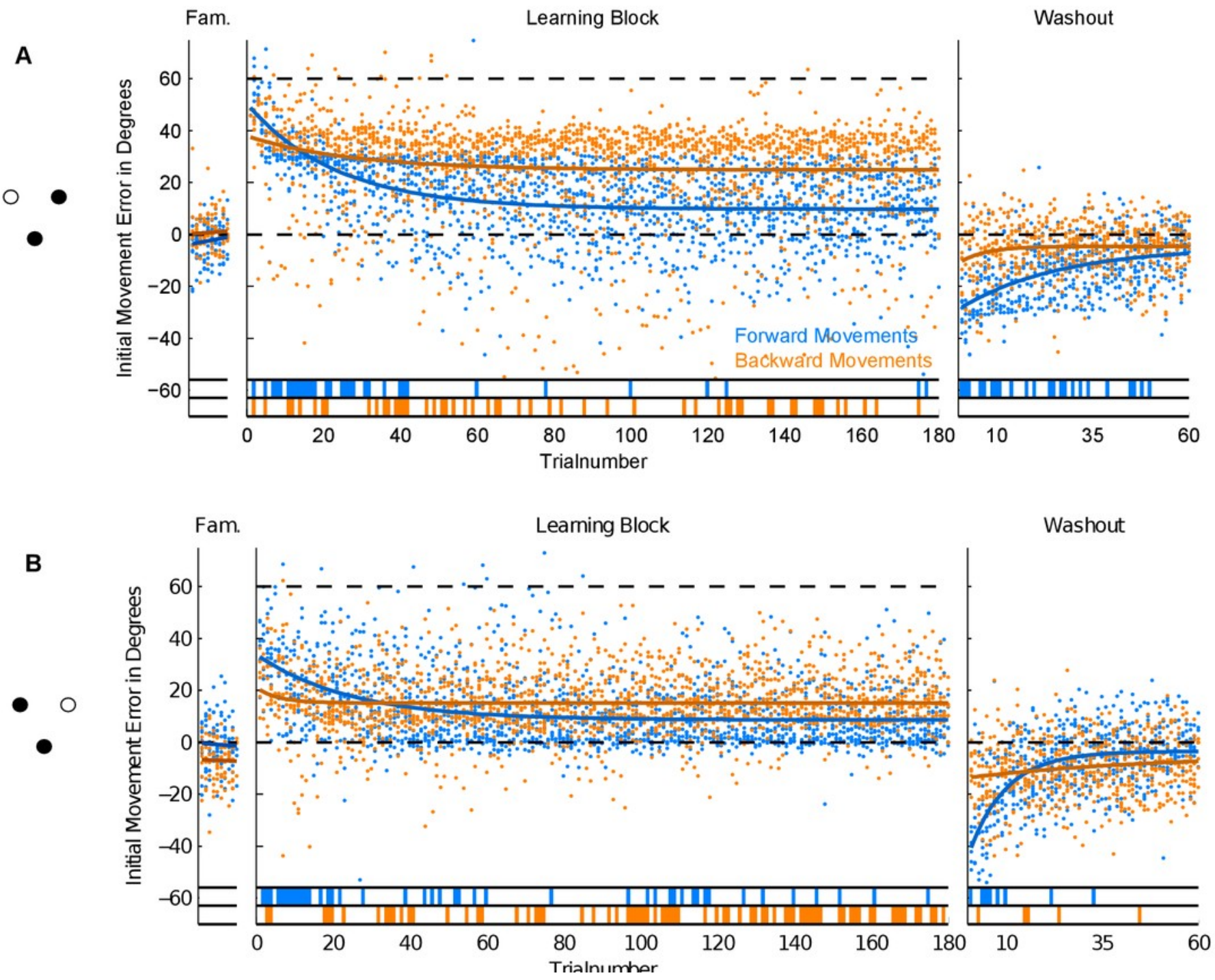


\section{Figure 8}

Comparison of Initial Movement Errors in the Beginning and in the End of Learning for Forward and Backward Movements.

(A) Comparing forward movements at the beginning and in the end of the learning block. (B) Comparing backward movements at the beginning and in the end of the learning block. (C) Comparing forward and backward movements at the beginning of the learning block. (D) Comparing forward and backward movements in the end of the learning block. Distributions were tested for significant differences with a ranksum test. P1 - Paradigm 1, all targets; P2a Paradigm 2, target at 60 degree; P2b - Paradigm 2, target at 240 degree; P3a - Paradigm 3, target at 60 degree; P3b - Paradigm 3, target at 120 degree. For Paradigm 1, the beginning of the learning block is taken as the first 50 trials, and the end of the learning block as the last 50 trials. In Paradigms 2 and 3, beginning means the first 15 trials, and end the last 15 trials. The height of the bars corresponds to the mean over trials. Error bars show the standard error of the mean (SEM) over trials and subjects. 

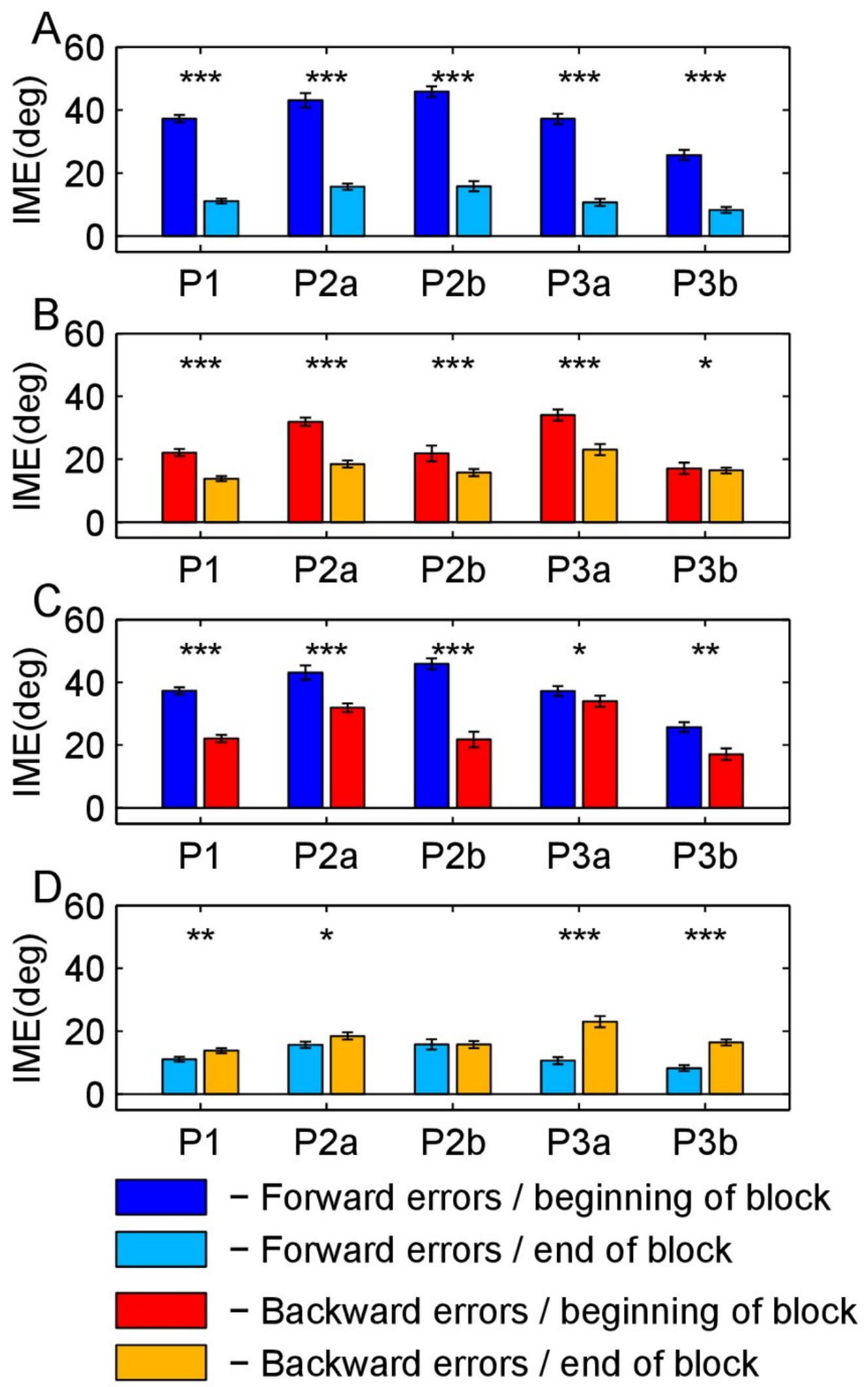

${ }^{*} p<0.05 ;{ }^{* *} p<0.01 ;{ }^{* * *} p<0.001$ 


\section{Figure 9}

Differences in Performance at the Beginning and the End of the Washout Block.

(A) Forward movements. (B) Backward movements. For color legend and significance levels see Figure 6. 

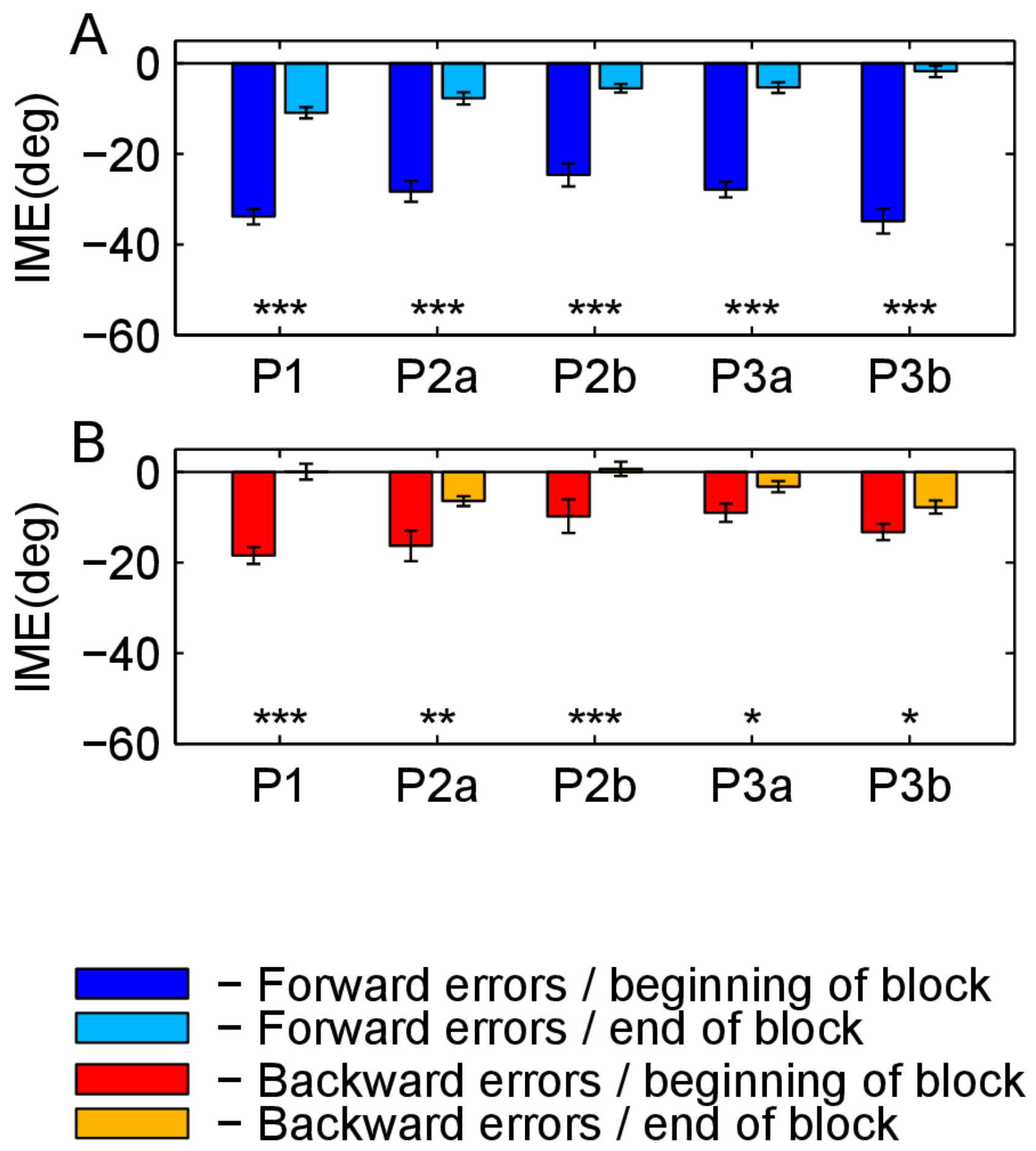


\section{Figure 10}

Differences Between Forward and Backward Learning Depending on the Learned Directions.

Each bar shown in the figure represents the difference between initial movement errors in forward and backward direction in the end of the learning block for one subjects. End of the learning block here is equivalent to the last 15 trials in the learning block (compare also methods and Figure 9). S1 to S10 are the 10 subjects in each of the paradigms (note that S1 in the second and the third paradigm was not the same person!). In A, differences are computed only for target P2a (paradigm two) and P3a (paradigm three), respectively. In B, differences are computed over all targets presented in the two paradigms (P2a, P2b, P3a, and P3b). The differences were significantly different between the two groups (ranksum test, $\mathrm{p}<0.05)$. 


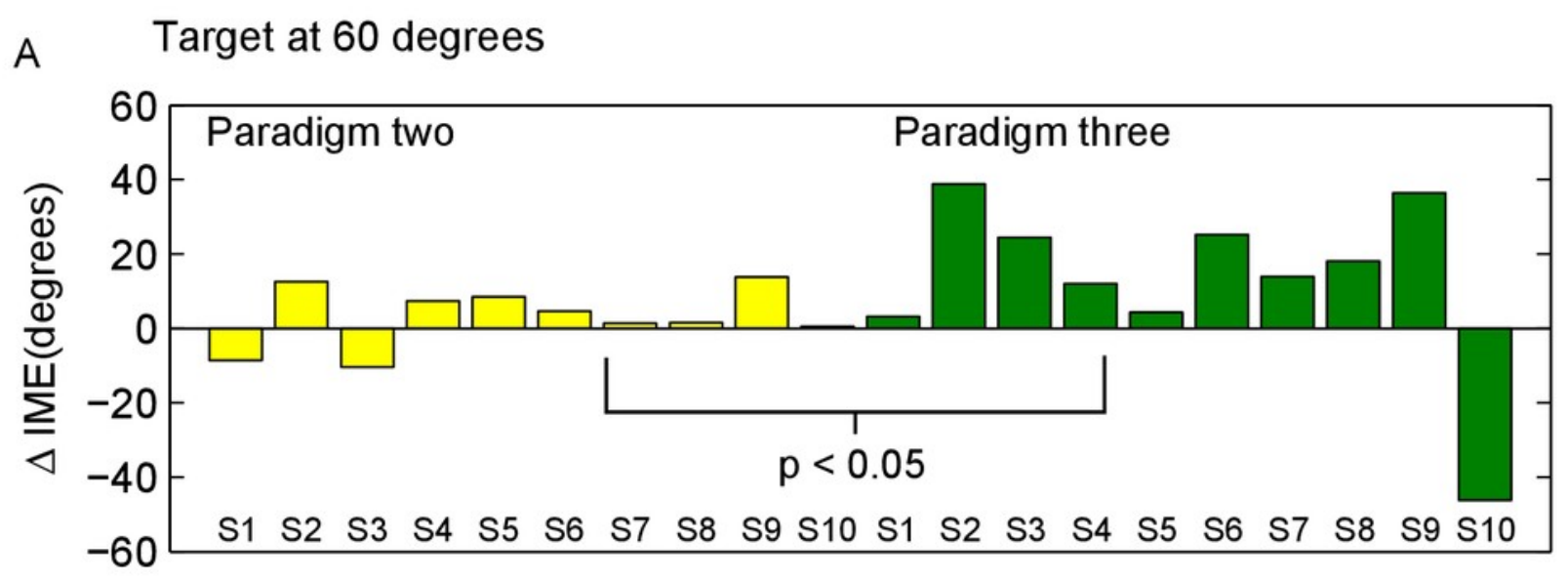

B Targets at 60,120 , and 240 degrees

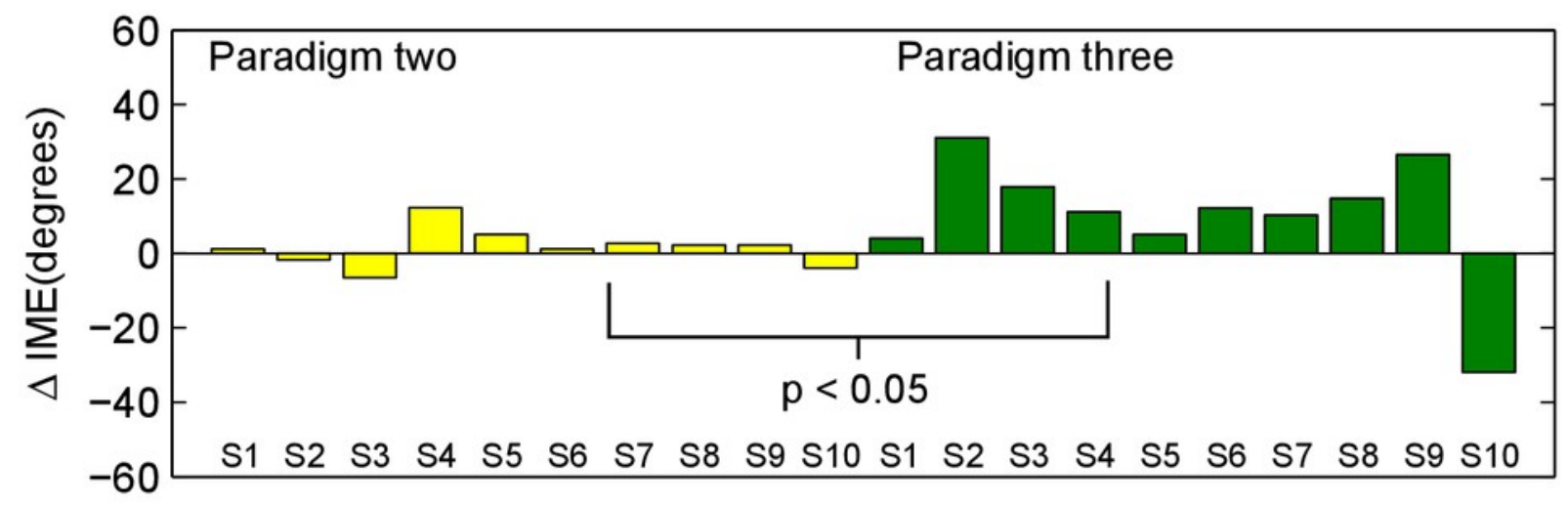

